\title{
BMP14 induces tenogenic differentiation of bone marrow mesenchymal stem cells in vitro
}

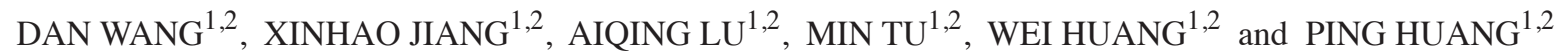 \\ ${ }^{1}$ Department of Orthopedics, Jinmen No. 2 People's Hospital; ${ }^{2}$ Department of Orthopedics, \\ Jingchu Center Hospital Affiliated to The Institute of Technology, Jingmen, Hubei 448000, P.R. China
}

Received March 16, 2017; Accepted May 24, 2018

DOI: $10.3892 /$ etm.2018.6293

\begin{abstract}
Bone marrow mesenchymal stem cells (BMSCs) are pluripotent cells, which have the capacity to differentiate into various types of mesenchymal cell phenotypes, including osteoblasts, chondroblasts, myoblasts and tendon fibroblasts (TFs). The molecular mechanism for tenogenic differentiation of BMSCs is still unknown. The present study investigated the effects of bone morphogenetic protein (BMP) 14 on BMSC differentiation in vitro. It was revealed that BMP14 significantly increased the expression of tendon markers (scleraxis and tenomodulin) at the mRNA and protein level, which led to the upregulation of sirtuin 1 (Sirt1) expression. The gain or loss of Sirt1 function may promote or inhibit tenogenic differentiation by deacetylating the peroxisome proliferator-activated receptor (PPAR) $-\gamma$. BMP14 also triggered the phosphorylation of c-Jun N-terminal kinase (JNK) and Smad1; overexpression of Sirt1 significantly increased the phosphorylation and knockdown of Sirt1 significantly decreased the phosphorylation. The inhibition of JNK and Smad significantly increased the acetylation of PPAR $\gamma$ and inhibited the expression of tenogenic differentiation markers. These results suggest that BMP14 may induce the tenogenic differentiation of BMSCs via the Sirt1-JNK/Smad1-PPAR $\gamma$ signaling pathway. The present study provided a cellular and molecular basis for the development of novel therapeutic strategies for tendon healing.
\end{abstract}

\section{Introduction}

Tendon and ligament injuries are among the most commonly encountered health problems, with about 16.4 million

Correspondence to: Professor Xinhao Jiang, Department of Orthopedics, Jingchu Center Hospital Affiliated to The Institute of Technology, 39 Xiangshan Road, Jingmen, Hubei 448000, P.R. China

E-mail: jiangxinhao0012@163.com

Key words: bone morphogenetic protein 14, bone marrow mesenchymal stem cell, tenogenic differentiation, Sirt1, peroxisome proliferator-activated receptor $\gamma, \operatorname{Smad} 1$ occurring every year in the United States (1). Cell-based therapies have been introduced with encouraging outcomes in preclinical evaluations and shows attractive future direction for clinical therapy of tendon injuries $(2,3)$. Bone marrow mesenchymal stem cells (BMSCs) are promising approaches for ligament and tendon reconstruction, and mostly used stem cell type to help tendon repair (4-6). In particular, a recent study found that the use of transforming growth factor (TGF)- $\beta 1$ and CTGF could initiated and maintained highly efficient tenogenesis of BMSCs (7).

Bone morphogenetic protein (BMP) 14, also known as cartilage-derived morphogenetic protein-1 (CDMP-1) and growth differentiation factor (GDF)-5, like other BMPs (BMP12 and BMP13), is a member of the TGF- $\beta$ family and has been shown to accelerate tendon healing in animal models $(8,9)$. The effect of BMP12 on induces tenogenic differentiation of adiposederived stromal cells (ASCs) and BMP13 on induces chondrogenic differentiation of murine mesenchymal progenitor cells have been well understooded $(10,11)$, but the function of BMP14 on tenogenic differentiation has not yet been investigated. Mounting evidences are showing that, BMP14 could increase tendon tensile strength in an achilles tendon injury rat model $(12,13)$. In a recent in vitro study, it was demonstrated that interposition of a multilayered collagen patch seeded with muscle-derived MSCs and BMP14 into the repair site enhanced flexor tendon healing compared with a similar patch using cells alone (14), but its molecular mechansim needs further confirmation.

Sirtuin 1 (Sirt1) is conserved protein NAD ${ }^{+}$-dependent histone deacylases, which is linked to various physiological process, including cell proliferation, apoptosis and inflammation $(15,16)$. Several studies have demonstrated important roles of Sirt1 in macrophages and chondrocyte differentiation $(17,18)$, and resveratrol induced Sirt1 activation promote sneuronal differentiation of human BMSCs (19). Furthermore, Sirt1 signaling pathway may be involved in rabbit flexor tendon repair and may be targeted for therapeutic intervention in flexor tendon injury (20), but its function role in tenogenic differentiation has not been reported. Previuos studies shows that, in HaCaT cells, UV radiation could induce Sirt1 down-regulation, ROS-mediated c-Jun N-terminal kinase (JNK) activation is involved in the process, and JNK inhibitor and antioxidant NAC could recover Sirt1 lost due to UV radiation (21). Sirt1 has recently been demonstrated to deacetylate 
Smad3 and Smad7 (22), these data indicating a direct function of Sirtl in the regulating JNK and Smad activity.

Many transcription factors play important roles in the BMSCs differentiation $(23,24)$. Including that, farnesoid $\mathrm{X}$ receptor $(\mathrm{FXR})$, a nuclear receptors, operates as a ligand-activated transcription factor (25), its activation stimulates BMSCs osteoblastic differentiation, whereas its inhibition leads to an adipocyte-like phenotype (26). Peroxisome proliferator-activated receptor (PPAR) $-\gamma$, another lignad-activated transcription factor belonging to the nucleus hoemone receptor superfamily (27), could inhibit BMSCs differentiation toward myofibroblasts (28).

Based on these observations, we suppose that whether BMP14 could induce BMSCs tendon differentiation and what molecular mechanism is involved. So, BMSCs were isolated, and the dose and time effects of BMP14 on BMSCs differentiation were examined targeting tendon markers and the molecular mechanism of BMP14 induced tendon differentiation was explored. Collectively, our research advances the current understandings of BMP14 induced tenogenic differentiation of BMSCs and provide a basis for future cellular and molecular approache for tendon tissue engineering and repair.

\section{Materials and methods}

Materials. $\alpha$-MEM medium, $0.25 \%$ trypsin, fetal bovine serum (FBS) and antibiotic/antimycotic solutions were obtained from Invitrogen (Thermo Fisher Scientific, Inc., Waltham, MA, USA). The anti-CD29 (ab52971), anti-CD44 (ab25340) anti-Sirt1 (ab110304), anti-PPAR $\gamma$ (ab45036), anti-Collagen type I (ab34710), anti-Collagen type III(ab7778), anti-tenomodulin (ab203676) and anti-SCX (ab58655) primary antibodies were obtained from Abcam (Cambridge, MA, USA). Anti-ERK1/2 (no. 4695), acetylated-lysine (no. 9441), anti-phospho-ERK1/2 (no. 4370), anti-JNK (no. 9258), anti-phospho-JNK (no. 4668), anti-Smad2/3 (no. 8685), anti-phospho-Smad2/3 (no. 11979), anti-Smad1 (no. 6944), anti-phospho-Smad1 (no. 5753) antibodies were purchased from Cell Signaling Technology, Inc., (Danvers, MA, USA), $\beta$-actin (A1978) monoclonal antibody was from Sigma-Aldrich (Merck KGaA, Darmstadt, Germany). CD44H monoclonal antibody (12-0444-82), CD73 monoclonal antibody (11-0739-42), CD90.1 Monoclonal antibody (A16370) and CD45 monoclonal antibody (11-0461-82) were purchased from eBioscience (Thermo Fisher Scientific, Inc.). BMP14 (SRP4580) was purchased from Sigma-Aldrich; Merck KGaA. LDN-193189 (S2618) and SP600125 (S1460) were obtained from Selleck (Shanghai, China).

Animals. All animal protocols were approved by the Institutions Animal Care and Use Committee of Jingchu Institute of Technology and complied with the guidelines of the Jingchu Institute of Technology's Regulations of Animal Experiments. Wistar-Kyoto (WKY) rats were obtained from Model Animal Research Center of Nanjing University. The rats were housed in a pathogen-free barrier facility with a $12 \mathrm{~h} \mathrm{light/dark} \mathrm{cycle} \mathrm{and} \mathrm{were} \mathrm{given} \mathrm{free} \mathrm{access} \mathrm{to} \mathrm{food} \mathrm{and}$ water. Rats were sacrificed under $\mathrm{CO}_{2}$ asphyxia (20\% volume of container per min) at 8-12 weeks of age and hind limbs were removed in preparation for bone marrow isolation.
BMSC isolation and identification. Bone marrow from the hind limbs of the WKY rat was isolated as previously described (29) and the expressions of cell surface markers on isolated BMSCs were measured using flow cytometry (30). Briefly, cells were collected and fixed in $4 \%$ paraformaldehyde, then stained with FITC-conjugated mouse anti-rat CD44, CD73, CD90, and CD45, and affinity purified mouse IgG was used as control. Fluorescence activated cell sorting was performed with FACS Calibur (BD Biosciences, Franklin Lakes, NJ, USA) and data were analyzed with FlowJo software (Tree Star, Ashland, OR, USA).

Reverse transcription-quantitative polymerase chain reaction $(R T-q P C R)$. After treatment, BMSCs were lysed with TRIzol (Thermo Fisher Scientific, Inc.) and total RNA was extracted according to the manufacturer's instructions. RNA concentration was determined by UV spectrophotometry (NanoDrop 2000; Thermo Fisher Scientific, Inc.). $1 \mu \mathrm{g}$ RNA was reversely transcribed into cDNA in a $20 \mu 1$ reaction using the M-MMLV Reverse Transcriptase with RNasin Ribonuclease Inhibitors (Promega Biotech Co., Ltd., Beijing, China). $1 \mu \mathrm{l}$ of the cDNA was used for subsequent RT-qPCR reactions with SYBR Green qPCR Master Mix (Biotool, Shanghai, China) according to the manufacturer's instructions using StepOnePlus Real-Time PCR System (Applied Biosystems; Thermo Fisher Scientific, Inc.). The PCR program was set as the follows: $95^{\circ} \mathrm{C}$ for $10 \mathrm{~min}$, followed by $95^{\circ} \mathrm{C}$ for $15 \mathrm{sec}, 60^{\circ} \mathrm{C}$ for $30 \mathrm{sec}$, and $72^{\circ} \mathrm{C}$ for $30 \mathrm{sec}$ for 40 cycles. The relative levels of target gene expression was analyzed using the comparative $\mathrm{Cq}\left(2^{-\Delta \Delta C q}\right)$ method (31). GAPDH was used as endogenous reference gene. The primer sequences used were: collagen I (forward: 5'-AAGGCCCACGGG GACCTGTT-3', reverse: 5'-GGGCCAGGCACGGAAACT CC-3'); collagen III (forward: 5'-AGCTGGACCAAAAGG TTGATG-3', reverse: 5'-GACCTCGTGCTCCAGTTAGC-3'); Scx (forward: 5'-AGAGACGGCGGCGAGAAC-3', reverse: 5'-AATCGCCGTCTTTCTGTCACG-3'); GAPDH (forward: 5'-CCTGGCCAAGGTCATCCAT-3', reverse: 5'-GAGTTG AGCAGCGTCTGGAT-3').

Fluorescent immunocytochemistry. BMSCs at passage 2 were initiated in 24-well chamber slides at a density of 2,500 cells per well in basal medium. At the end of culture, cells were fixed in $4 \%$ paraformaldehyde overnight, rinsed in phosphate-buffered saline (PBS) and blocked using 10\% sheep serum in PBS with 0.1\% Triton X-100. CD29, CD44 and Scx primary antibodies were diluted at 1:200 and 1:100 respectively and incubated overnight at $4^{\circ} \mathrm{C}$, then Donkey Anti-Rabbit IgG H\&L (Alexa Fluor ${ }^{\circledR}$ 647) (ab150075) and Donkey Anti-Rabbit IgG H\&L (Alexa Fluor ${ }^{\circledR}$ 488) (ab150073) were used at a 1:1,000 dilution for $1 \mathrm{~h}$ in a solution containing $1 \%$ bovine serum albumin (BSA). After extensive washes in PBS, slides were mounted by using SlowFade ${ }^{\circledR}$ Gold Anti fade Mountant with DAPI (S36942; Thermo Fisher Scientific, Inc.), and observed by fluorescence microscopy (Olympus IX81; Olympus Corporation, Tokyo, Japan).

Induction of BMSC differentiation. To examine the effect of BMP14 on tenogenic differentiation, BMSCs were treated with BMP14 for different dose and different time. 
The dose of BMP14 was chosen based on the results from this study, which resulted in the strongest tenogenic effects on BMSCs among all the doses and time examined. For western blot assay, BMSCs were cultured in 6-well plates at a density of $2 \times 10^{5}$ cells per well, then cells were treated with either BMP14 $(50 \mathrm{ng} / \mathrm{ml})$ for the indicated periods in duplicates. In cases when the inhibitor LDN-193189 $(1 \mu \mathrm{M})$ or SP600125 $(1 \mu \mathrm{M})$ was applied, the cells were pretreated with either of the drugs for $15 \mathrm{~min}$ before the addition of BMP14.

Western blot assay. BMSCs cells were collected, lysed, and subjected to protein extraction with RIPA buffer. Twenty-five micrograms of total lysate were separated on sodium dodecyl sulfate-polyacrylamide gel electrophoresis (SDS-PAGE) gels and then transferred onto nitrocellulose membranes. Specific monoclonal anti-Sirt1 (1:1,000 dilution), anti-Collagen Type I (1:1,000 dilution), anti-Collagen Type III (1:1,000 dilution) anti-tenomodulin (1:1,000 dilution) and Anti-SCX antibody (1:1,000 dilution) primary antibodies (Abcam) and anti-ERK1/2 (1:1,000 dilution), anti-phospho-ERK1/2 (1:1,000 dilution), anti-JNK (1:1,000 dilution), anti-Phospho-JNK (1:1,000 dilution), anti-Smad2/3 (1:1,000 dilution), anti-phospho-Smad2/3 (1:1,000 dilution), anti-Smad1 (1:1,000 dilution), anti-phospho-Smad1 (1:1,000 dilution) were used, and anti-rabbit HRP conjugated secondary antibody (no. 7074; Cell Signaling Technology, Inc., Danvers, MA, USA) was used. West Pico Chemiluminescent (Pierce; Thermo Fisher Scientific, Inc.) was used as the substrate to visualize protein bands, which were quantified using densitometry image analysis software (Image Master VDS; Pharmacia Biotech, Uppsala, Sweden). Normalization was made against $\beta$-actin (1:3,000 dilution) expression.

IP-western blot analysis. Cell lysate was incubated overnight at $4^{\circ} \mathrm{C}$ with PPAR $\gamma$ antibody and then $2 \mathrm{~h}$ with protein $\mathrm{A} / \mathrm{G}$ beads (B23012; Biotool). After washing with PBS, bound proteins were separated in $10 \%$ SDS-PAGE, transferred to polyvinylidene fluoride (PVDF) membrane, blocked with 5\% milk in TBST, and incubated with acetylated-lysine, Sirt1 or PPAR $\gamma$ antibodies overnight at $4^{\circ} \mathrm{C}$. After washing with TBST, the membrane was incubated at room temperature for $1 \mathrm{~h}$ with anti-mouse antibody conjugated with horseradish peroxidase (HRP). Signals were revealed by enhanced chemiluminescence (ECL) followed by exposure.

Lentivirus infection. BMSC cells were infection with lentivirus vectors expressing Sirt1 or shSirt1 which were obtained from GenePharm Co., Ltd. (Shanghai, China). ShRNA sequences were as follows: Shsirt-1: GAAGTGCCTCAGATATTAA, Shscramble: GCGCGCTTTGTAGGATTCG.

Statistics. Data were presented as means \pm standard error mean of three independent experiments. Data between groups were analyzed by Student's t-test or one-way analysis of variance followed by Bonferroni-Dunn multiple comparison. All statistical analyses were conducted using SPSS version 16.0 software (SPSS, Inc., Chicago, IL, USA). P<0.05 was considered to indicate a statistically significant difference.

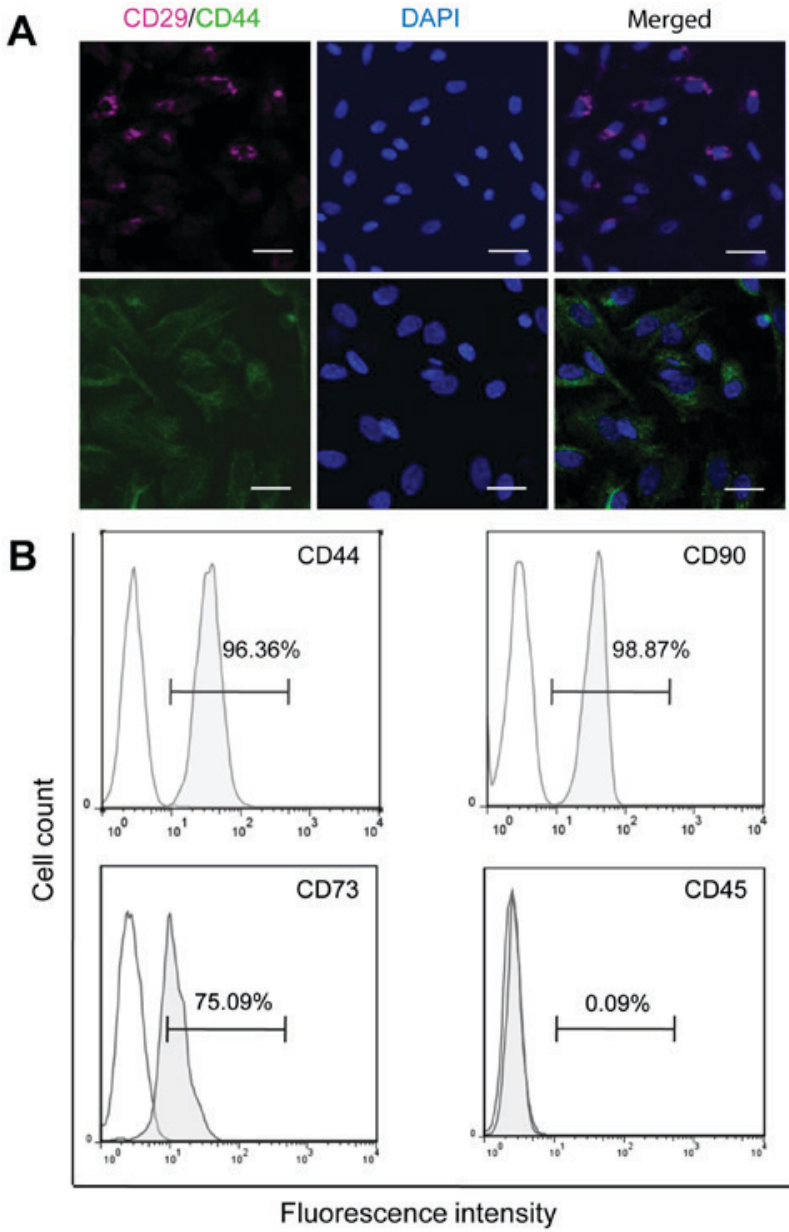

Figure 1. Characterization of BMSCs. (A) Representative images show immunofluorescent staining of CD29 and CD44 on passage 2 BMSCs. Scale bars, $50 \mu \mathrm{m}$. Pink, CD29; green, CD44; blue, DAPI. (B) Flow cytometry identified rat BMSC-positive markers CD44, CD90 and CD73, but not the marker CD45 on the surface of the cells. CD, cluster of differentiation; BMSC, bone marrow mesenchymal stem cell.

\section{Results}

Characterization of BMSCs. There are two kinds of stem cells in the bone marrow: Mesenchymal and hematopoietic stem cells (32). To initiate the characterization of BMSCs, immunofluorescent staining was used to identify the BMSCs with surface markers CD29 and CD44 (33). As shown in Fig. 1A, CD29 and CD44 were both positively detected on BMSCs at passage 2 .

The expression of the cell markers specific for mesenchymal and hematopoieticstem cells was analyzed with flow cytometry. All analyzed cells were positive for mesenchymal cell markers CD44, CD73 and CD90, but negative for hematopoietic cell marker CD45 (Fig. 1B), confirming the identity of isolated BMSCs.

BMP14 induce tenogenic gene expression in BMSCs. The dose and time effects of BMP14 on BMSC differentiation were further investigated using RT-qPCR assay targeting the Scx and the tendon matrix genes collagen I and collagen III. Scx is a tendons and ligaments specific marker (34) which regulates TNMD expression (35), and TNMD is required 
A

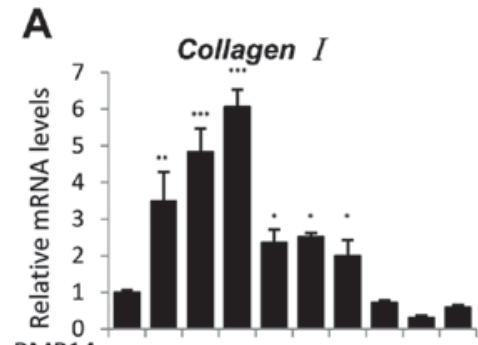

$\begin{aligned} & \text { BMP14 } \\ & (\mathrm{ng} / \mathrm{ml}) \\ & \text { Day(s) }\end{aligned}$
Day(s $\frac{1050}{2} \frac{102050}{5} \frac{102050}{7}$
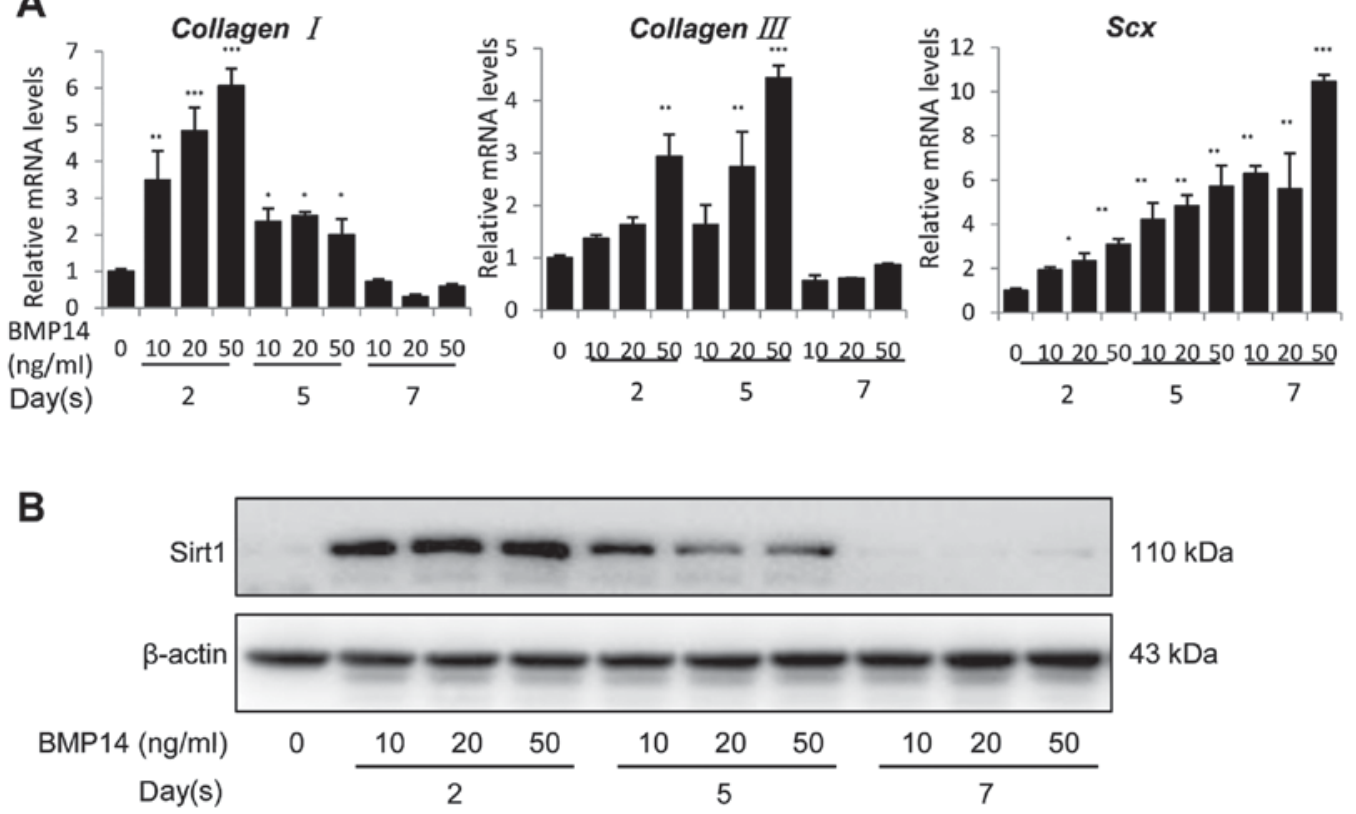

Figure 2. BMP14 induces tenogenic gene expression in BMSCs. (A) The mRNA levels of collagen I, collagen III and Scx in BMSCs treated with BMP14 at 2, 5 and 7 days. (B) The protein expression of Sirt1 in BMSCs increased following treated with 10, 20 and 50 ng/ml BMP14 at 2, 5 and 7 days. The data are presented as the mean \pm standard error of the mean and expressed as the fold-change relative to the level of the control. ${ }^{*} \mathrm{P}<0.05$, ${ }^{* *} \mathrm{P}<0.01$ and ${ }^{* * * *} \mathrm{P}<0.001 \mathrm{vs}$. the 0 group. BMSC, bone marrow mesenchymal stem cell; Scx, scleraxis; BMP, bone morphogenetic protein; Sirt1, sirtuin 1.

for tendon fibroblasts (TFs) proliferation and tendon maturation (36). Our results showed that BMP14 dramatically increased collagen $I$ expression in mRNA levels by up to 3.5-6-fold at day 2, but decreased to $\sim 2.5$-fold at day 5 and $\sim 0.75$-fold at day 7 (Fig. 2A left). $50 \mathrm{ng} / \mathrm{ml} \mathrm{BMP14}$ also caused a substantial increase in collagen III expression (Fig. 2A middle) at day 2 and day 5, it also increased $S c x$ mRNA expression in BMSCs by up to 11-fold in a dose and time dependent manner (Fig. 2A right). Furthermore, BMP14 promotes the Sirt1 protein content in BMSCs at day 2 but dramatically decreased at day 5 and day 7 (Fig. 2B). This may response to the reduced differentiation potential after long time culture (37). These results suggest that BMP14 regulates the differentiation of BMSCs by a mechanism involving the expression of Sirt1.

Sirtl gain of function promotes BMP14 induced tenogenic differentiation of BMSCs. Overexpression of Sirt1 was identified using western blotting and qPCR as shown in Fig. 3A, and Sirt1 could increased BMP14 induced tendon marker gene collagen I, collagen III and Scx mRNA expression in BMSCs (Fig. 3B), indicating that Sirt1 overexpression could promote BMP14 induced tenogenic differentiation of BMSCs. Western blot assay showed that Sirt1 could also increase the tendon marker collagen I, TNMD and Scx protein expression in BMSCs caused by BMP14 (Fig. 3C), Sirtl overexpression shows increased tendon markers mRNA and protein expression than control group, sirt1 combine with BMP14 shows more increased tendon marker levels than BMP14 or Sirt1 group. Immunofluorescence staining using Scx antibody shows the samilar result as western blot assay, and the fluorescence intensity was quantified (Fig. 3D). Co-immunoprecipitation (Co-IP) results showed that BMP14 treatment and Sirt1 overexpression decreased PPAR $\gamma$ acetylation levels in BMSCs, BMP14 plus Sirt1 could further decrease the acetylation levels (Fig. 3E).

Knockdown Sirtl could inhibit the BMP14 induced tenogenic differentiation of BMSCs. Knockdown of Sirt1 was performed using shSirt1 lentivirus infection for $48 \mathrm{~h}$ and evaluated with western blot and real-time PCR assay (Fig. 4A). Knockdown Sirt1 could decreased BMP14 induced tendon marker gene collagen I, collagen III and Scx mRNA expression in BMSCs (Fig. 4B and C). Western blot assay showed that knockdown Sirt1 could also decrease the tendon marker collagen I, TNMD and Scx protein expression in BMSCs caused by BMP14 (Fig. 3C), only knockdown sirt1 shows decreased tendon markers mRNA and protein expression than control group. Shsirt1 combine with BMP14 shows increased tendon marker levels than shsirt1, but decreased levels than BMP14. Immunofluorescence staining using Scx antibody shows the similar result as western blot assay (Fig. 4D), and the fluorescence intensity was quantified.

Co-IP results showed that BMP14 treatment decreased PPAR $\gamma$ acetylation levels in BMSCs, but knockdown Sirt1 could increase the acetylation levels (Fig. 4E). Since Sirt1 is a protein deacetylase implicated in the regulation of metabolic activity, so the results of Sirt1 regulate the acetylation levels of PPAR $\gamma$ is consistence with the previous findings (38).

BMP14 induced tenogenic differentiation of BMSCs via the JNK and Smadl pathway in Sirt1-dependent manner. BMPs are known to activate both Smad pathways and non-Smad pathways such as mitogen-activated protein kinase (MAPK) pathway $(39,40)$. As shown in Fig. 5A, BMP14 induced increased phosphorylation of Smad1 and JNK, but has no effect on the phosphorylation of p38 and $\operatorname{Smad} 2 / 3$. We next asked whether Sirtl could affect the activation of Smad and 
A

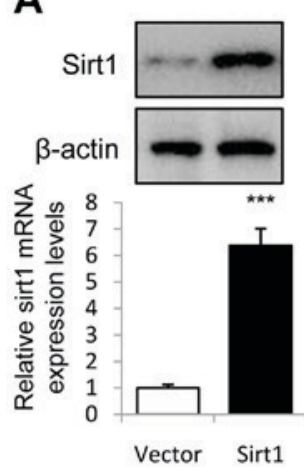

B

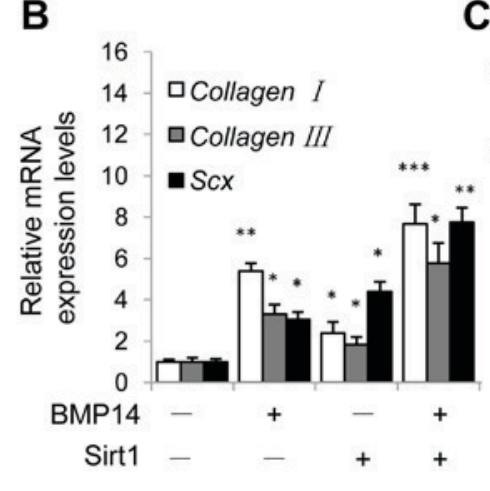

C

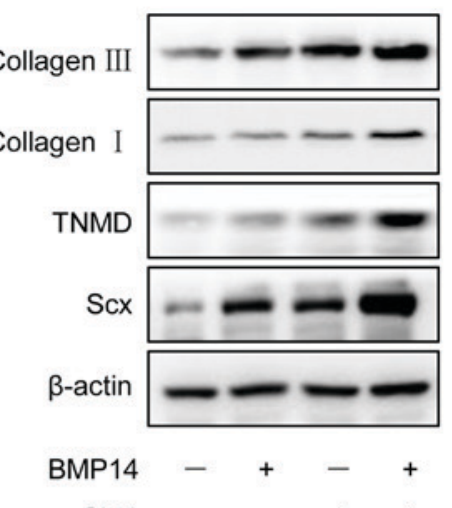

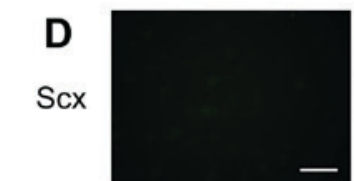
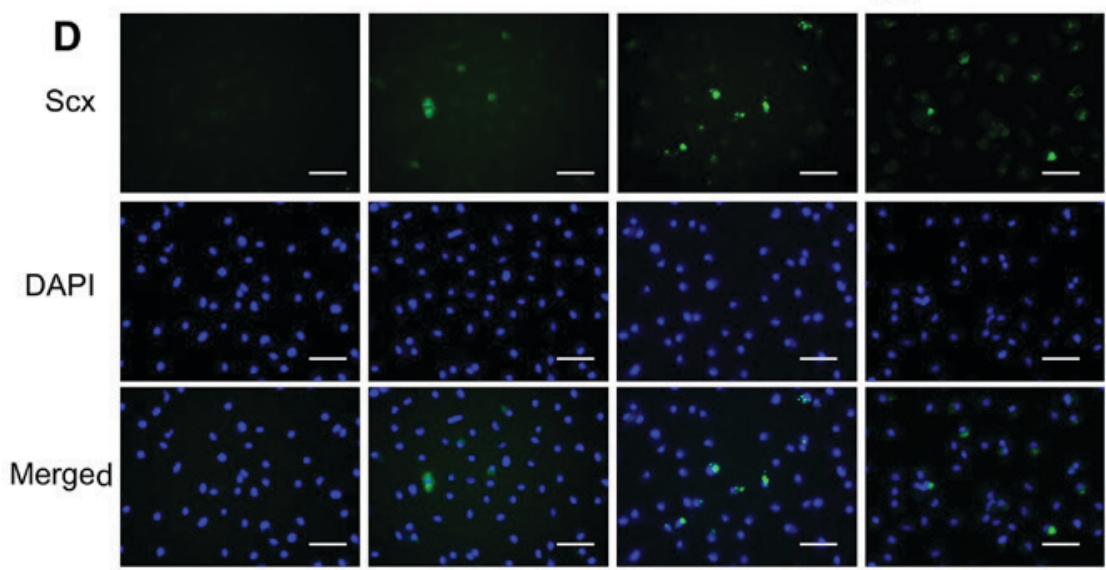

BMP14
Sirt1

$+$
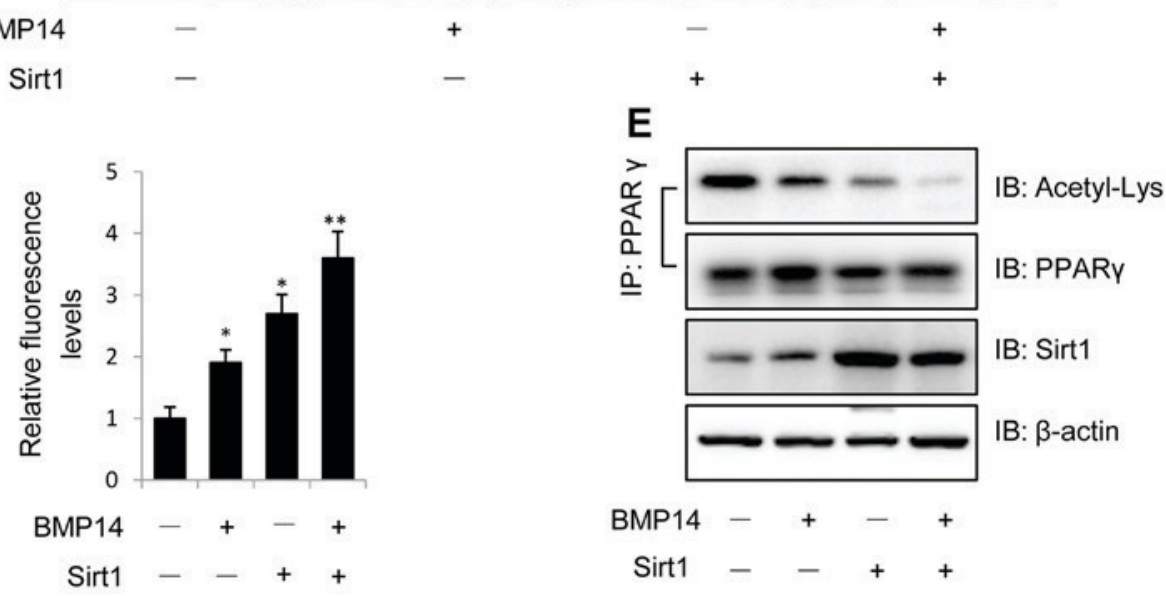

Figure 3. Overexpression of Sirt1 promotes BMP14 induced tenogenic differentiation of BMSCs. (A) Sirt1 protein and mRNA levels were determined by western blot and RT-qPCR at $48 \mathrm{~h}$ post infection with the vector control or Sirt1 lentivirus. $\beta$-actin was used as the loading control. (B) mRNA expression of collagen I, collagen III and Scx and (C) protein expression of collagen I, collagen III, TNMD and Scx were detected using RT-qPCR and western blot analysis with or without $50 \mathrm{ng} / \mathrm{ml}$ BMP14 treatment for $48 \mathrm{~h}$. (D) Fixed BMSCs were stained with Scx antibodies and Alexa Fluor 488 goat anti-rabbit secondary antibodies (green) and DAPI (blue). Scale bars, $200 \mu \mathrm{m}$. (E) PPAR $\gamma$ acetylation levels in BMSCs were analyzed using immunoprecipitation with anti-PPAR $\gamma$ antibodies and immunoblotting with anti-Acetyl-Lys and anti-PPAR $\gamma$ antibodies. The total cell lysate was immunoblotted with anti-sirt1 and anti- $\beta$-actin antibodies. Each bar represents the mean \pm standard error of the mean. The results were repeated in three independent experiments. ${ }^{*} \mathrm{P}<0.05,{ }^{* *} \mathrm{P}<0.01$ and ${ }^{* * * *} \mathrm{P}<0.001$ vs. the control. RT-qPCR, reverse transcription-quantitative polymerase chain reaction; BMP, bone morphogenetic protein; BMSC, bone marrow mesenchymal stem cell; Sirt1, sirtuin 1; Scx, scleraxis; TNMD, tenomodulin; PPAR $\gamma$, peroxisome proliferator-activated receptor $\gamma$; IP, immunoprecipitation; IB, immunoblotting.

MAPK pathway in BMSCs induced by BMP14. To answer this question, lentivirus overexpress Sirt1 and shSirt1 were used. Interestingly, overexpression of Sirt1 in BMSCs could increase the phosphorylation of Smad1 and JNK, and knockdown Sirt1 could significantly decrease the phosphorylation levels (Fig. 5A). Furthermore, TGF- $\beta /$ Smad pathway inhibitor LDN-193189 and JNK inhibitor SP600125 were used to detect whether can block the BMP14 decresed the acetylation of PPAR $\gamma$ effect. As we expected, $1 \mu \mathrm{M} \mathrm{LDN}-193189$ and SP600125 both can increase the acetylation of PPAR $\gamma$ (Fig. 5C).

RT-qPCR and western blot assay demonstrated that Sirt1 promote Smad1 and JNK mediated Scx mRNA and protein expression and collagen I, collagen III protein levels, however, BMP14 treatment had similar proportional increasement between vector and Sirt1 forced expression, but LDN-193189 

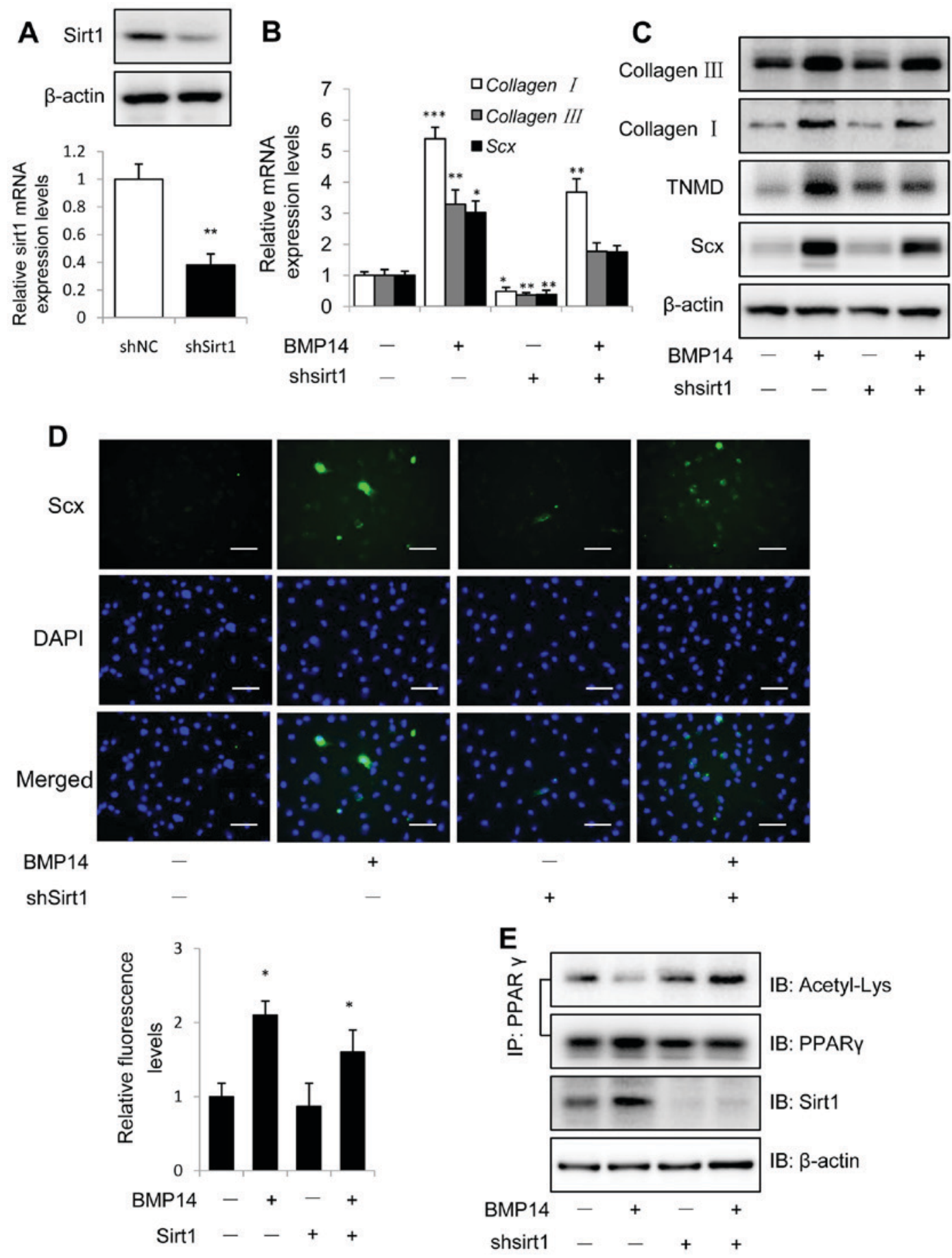

Figure 4. Knockdown Sirt1 inhibits BMP14 induced tenogenic differentiation of BMSCs. (A) Sirt1 protein and mRNA levels were determined by western blot analysis and RT-qPCR at $48 \mathrm{~h}$ post infection with shNC or shSirt1 lentivirus. $\beta$-actin was used as the loading control. (B) The mRNA expression collagen I, collagen III and Scx and (C) the protein expression of collagen I, TNMD and Scx were detected in BMSCs infected with shNC or shSirt1 using RT-qPCR and western blot analysis, respectively with or without $50 \mathrm{ng} / \mathrm{ml}$ BMP14 treatment for $48 \mathrm{~h}$. (D) Fixed BMSCs were stained with Scx antibodies and Alexa Fluor 488 goat anti-rabbit secondary antibodies (green) and DAPI (blue). Scale bar, $200 \mu \mathrm{m}$. (E) PPAR $\gamma$ acetylation in BMSCs was analyzed using immunoprecipitation with anti-PPAR $\gamma$ antibodies and immunoblotting with anti-Acetyl-Lys and anti-PPAR $\gamma$ antibodies. The total cell lysate was immunoblotted with anti-sirt1 and anti- $\beta$-actin antibodies. Each bar represents the mean \pm standard error of the mean. The results were repeated in three independent experiments. "P<0.05, ${ }^{* *} \mathrm{P}<0.01$ and ${ }^{* * * *} \mathrm{P}<0.001$ vs. the control. BMSC, bone marrow mesenchymal stem cell; Sirt1, sirtuin 1; NC, negative control; sh, short hairpin; RT-qPCR, reverse transcription-quantitative polymerase chain reaction; BMP, bone morphogenetic protein; Sirt1, Sirtuin1; Scx, scleraxis; TNMD, tenomodulin; PPAR $\gamma$; peroxisome proliferator-activated receptor $\gamma$; IP, immunoprecipitation; IB, immunoblotting.

or SP600125 decreased the Scx mRNA and protein levels and collagen I, collagen III protein expression (Fig. 5B), the lentivirus knockdown Sirtl showed the same effect. These data suggest that BMP14 promote effect on tenogenic differentiation markers through Smad1 and JNK pathway.

\section{Discussion}

The current study investigated the effects and molecular mechanism of BMP14 on BMSC tenogenic differentiation, thus further demonstrating its role as a tenogenic cue. Besides, 
A

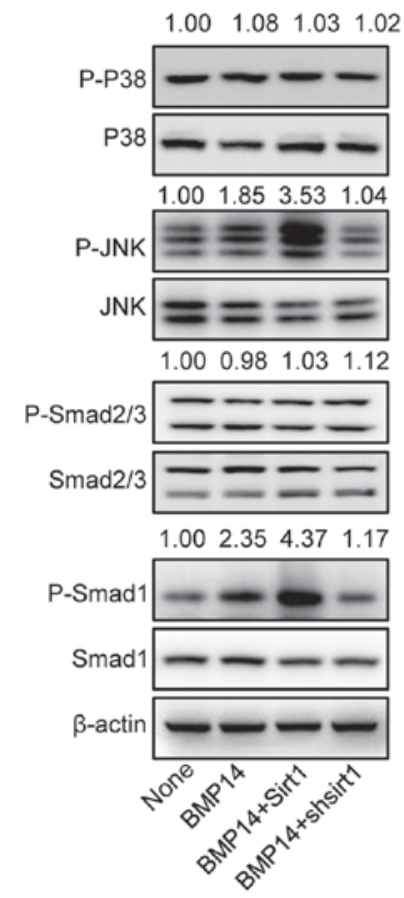

B
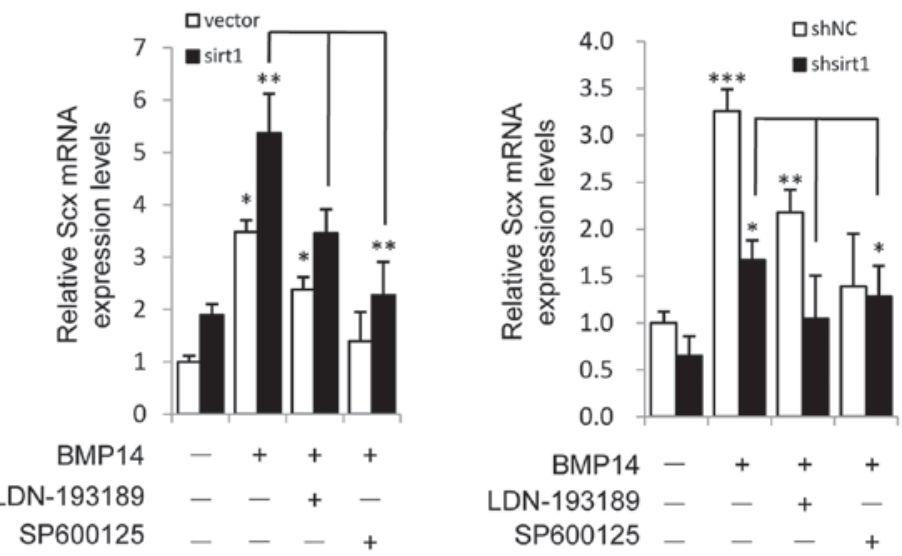

C
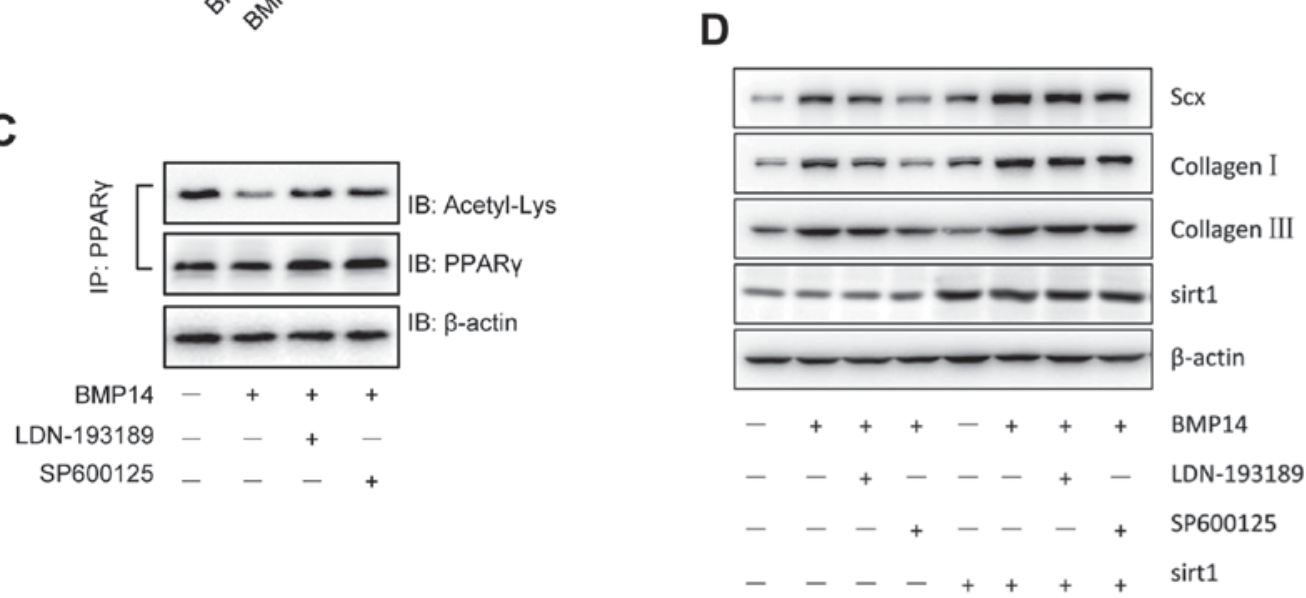

Figure 5. BMP14 induces tenogenic signaling in BMSCs via the JNK and Smad1 pathway in a Sirt1-dependent manner. (A) BMSC cells were infected with Sirt1 overexpressing or shSirt1 lentivirus. Following treatment with $50 \mathrm{ng} / \mathrm{ml}$ BMP14, the cell extracts were blotted with anti-P-p38, p38, P-JNK, JNK, P-smad2/3, smad2/3, P-smad1 and smad1 antibodies. Quantitative analysis of the blots was performed using ImageJ software and the relative ratios are indicated above the blots. (B) mRNA levels of Scx were detected using RT-qPCR. (C) BMSC cells were treated with $50 \mathrm{ng} / \mathrm{ml}$ BMP14 plus $1 \mu$ M LDN-193189 and SP600125. Following $48 \mathrm{~h}$ the cells were harvested and cell extracts were immunoprecipitated with PPAR $\gamma$ antibodies; the blots were immunoblotted with acetyl-lys and PPAR $\gamma$ antibodies. The total cell lysate was immunoblotted with anti- $\beta$-actin antibody. $\beta$-actin was used as the control. BMSC cells were treated with $50 \mathrm{ng} / \mathrm{ml}$ BMP14 with or without $1 \mu \mathrm{M}$ LDN-193189 or SP600125. (D) The protein expression of Scx, collagen I, collagen III and Sirt1 in BMSC cells were detected by western blot analysis. $\beta$-actin was used as the loading control. The data are presented as the mean \pm standard error of the mean. $\mathrm{P}<0.05,{ }^{* *} \mathrm{P}<0.01$ and ${ }^{* * *} \mathrm{P}<0.001$. RT-qPCR, reverse transcription-quantitative polymerase chain reaction; BMP, bone morphogenetic protein; Sirt1, sirtuin 1; BMSC, bone marrow mesenchymal stem cell; sh, short hairpin; p-, phosphorylated; JNK, c-Jun N-terminal kinase; PPAR $\gamma$, peroxisome proliferator-activated receptor $\gamma$; Scx, scleraxis; IP, immunoprecipitation; IB, immunoblotting.

we found that BMP14 promotes BMSCs differentiation in Sirt1-dependent manner via JNK and Samd1 pathway. Promoting BMP14 induced BMSCs tenogenic differentiation with a longterm view in therapeutic strategies for improving tendon to bone healing (41).

BMP14 has been shown to play a role in a variety of musculoskeletal processes, including joint formation (42), endochondral ossification (43), tendon and ligament maintenance and repair $(44,45)$ and even brown adipogenesis in systemic energy expenditure (46). Previous studies showed that both BMP12 and BMP14 were capable of inducing tendon marker gene expression in ASCs, but BMP12 was marginally more potent and selective than BMP14 (10). But the effect of BMP14 on BMSCs was poorly understood. So, in the present study, we propose a mechanism by which BMP14 promotes Sirt1-mediated PPAR $\gamma$ deacetylation might contribute to this process.

Firstly, we observed that $50 \mathrm{ng} / \mathrm{ml} \mathrm{BMP14}$ had a tenogenic differentiation effect on BMSCs in a dose- and time-dependent manner when measured at day 2. BMP14 upregulates Sirt1 both at mRNA and protein levels compared to untreated BMSCs. Several studies have demonstrated important roles of Sirt1 in macrophages and chondrocyte differentiation $(17,18)$, but its function role in tenogenic differentiation role has not been reported. 
To comprehensively understand the effect of Sirt1 in tenogenic differentiation, we performed in vitro western blot and real-time PCR assays to identify the tenogenic markers expression of BMSCs cells with over-expression or knockdown of Sirt1. Our results show that Sirtl promotes cell tenogenic differentiation. Consistently, Sirtl knockdown decrease that process of BMSCs cells, implying that Sirt1 functions as mediator in the tenogenic differentiation. As Sirt1 is a NAD-dependent deacetylase that removes acety group from proteins and modulating protein activity, Co-IP assay was further used to reveal that the PPAR $\gamma$ deacetylation may be the reason for the differentiation-promoting effect of Sirt1.

PPAR $\gamma$ is mainly known to regulate metabolism, immune responses and cellular proliferation. Recently, its functional role in adipocyte differentiation via Sirt1 has been investigated (38). Here, it is confirmed to have a similar effect in tenogenic differentiation. The deacetylation levels of PPAR $\gamma$ by BMP14 treatment was even reduced by overexpression of Sirt1, indicating that Sirt1 could regulate the deacetylation of PPAR $\gamma$ in BMSCs differentiation.

BMPs comprise the largest subgroup of the TGF superfamily and also known to activate both Smad pathways and MAPK pathways $(47,48)$. But in our study only the phosphorylation of JNK and Smad1 were observed, indicating that ERK and $S m a d 2 / 3$ are not involved in this process. As previously reported, Smad and MAPK pathways were downstream of Sirt1, the activation of Sirt1 could increase the phosphorylation of Smad and MAPK pathway (49,50). Taken together with the observation that BMP14 can activate Sirt1 and deacetylate PPAR $\gamma$, we hypothesis that BMP14 decreased the acetylation of PPAR $\gamma$ through Smad and JNK pathway. Interestingly, the treatment with Smad inhibitor LDN-193189 or JNK inhibitor SP600125 could increase the PPAR $\gamma$ acetylation, and affect the Sirt1 regulated tenogenic differentiation markers expression. We hypothesize that, in BMSCs, BMP14 induced the deacetylation of PPAR $\gamma$ through activation of Sirt1-Smad1/JNK pathway, deacetylated PPAR $\gamma$ further promote the tendon differentiation of BMSCs.

In 2004, Wang et al (51) noted that the in vitro applications of BMP-14 induced cellular activation/proliferation in primary intervertebral discs (IVD) cells, promoting collagen synthesis. But higher doses produced inflammatory reactions, indicating the positive effect of GDF-5 may depend on dosing, and that multiple treatments can cause side effect like catabolic and inflammation (51). It has been shown that growth factors such as TGF- $\beta$, insulin-like growth factor-1 (IGF-1) and BMP2 can induce differentiation of BMSCs into chondrocytes under appropriate conditions, and has been successfully applied to tissue engineering and transgenic technology to help damage the repair of cartilage $(52,53)$. BMP14 is one of the important regulators of cartilage formation and skeletal development in the body. It can promote the proliferation, adhesion, aggregation and differentiation of articular chondrocytes (ACS) and mesenchymal cartilage cells (MSCs) in the early stage of cartilage formation (54). The high cell induced by BMP14 form micromass, which mimics prechondrogenic cellular condensation which occurs during embryonic development, and is widely used as a favorable environment for chondrogenesis (55).

Taken together, our study demonstrated BMP14 as a potent tenogenic differentiation cue, shows the potential to induce
BMSCs to form TF-like cells, and further elucidated the signaling pathway leading to tenogenesis of BMSCs. These findings provide a cellular and molecular basis for developing novel therapeutic strategies for tendon healing, and throw light on the usage of BMP14 in the clinical application, despite the side effect and risk need further investigation.

\section{Acknowledgements}

The authors would like to thank Dr. Qi Zhang (China Pharmaceutical University, Nanjing, China) for their help with the isolation and identification of BMSCs from rats, and also Dr. Mingming Chen (Jingchu Center Hospital Affiliated to the Institute of Technology, Jingmen, China) for proof-reading and editing the manuscript.

\section{Funding}

No funding was received.

\section{Availability of data and materials}

The datasets used and/or analyzed during the current study are available from the corresponding author on reasonable request.

\section{Authors' contributions}

DW and AL performed the immunoblotting and luciferase analysis, analyzed the results and wrote part of the manuscript. MT performed RT-qPCR and wrote part of the manuscript. WH supervised the study, analyzed the luciferase assays and wrote part of the manuscript. PH and XJ designed and directed the experiments and wrote the manuscript. All authors have read and approved the final manuscript.

\section{Ethics approval and consent to participate}

All animal protocols were approved by the Institutions Animal Care and Use Committee of Jingchu Institute of Technology (Hubei, China) and complied with the guidelines of the Jingchu Institute of Technology's Regulations for Animal Experiments.

\section{Consent for publication}

Not applicable.

\section{Competing interests}

The authors declare that they have no competing interests.

\section{References}

1. Furumatsu T, Shimamura Y and Nishida K: Analysis of musculoskeletal systems and their diseases. Pathology and treatment for injuries of the tendon and ligament. Clin Calcium 25: 1205-1211, 2015.

2. Omae H, Sun YL, An KN, Amadio PC and Zhao C: Engineered tendon with decellularized xenotendon slices and bone marrow stromal cells: An in vivo animal study. J Tissue Eng Regen Med 6: 238-244, 2012.

3. Gaspar D, Spanoudes K, Holladay C, Pandit A and Zeugolis D: Progress in cell-based therapies for tendon repair. Adv Drug Deliv Rev 84: 240-256, 2015. 
4. Hankemeier S, Keus M, Zeichen J, Jagodzinski M, Barkhausen T, Bosch U, Krettek C and Van Griensven M: Modulation of proliferation and differentiation of human bone marrow stromal cells by fibroblast growth factor 2: Potential implications for tissue engineering of tendons and ligaments. Tissue Eng 11: 41-49, 2005

5. Chen HS, Chen YL, Harn HJ, Lin JS and Lin SZ: Stem cell therapy for tendon injury. Cell Transplant 22: 677-684, 2013.

6. Zhao C, Chieh HF, Bakri K, Ikeda J, Sun YL, Moran SL, An KN and Amadio PC: The effects of bone marrow stromal cell transplants on tendon healing in vitro. Med Eng Phys 31: 1271-1275, 2009.

7. Yin Z, Guo J, Wu TY, Chen X, Xu LL, Lin SE, Sun YX, Chan KM, Ouyang H and Li G: Stepwise differentiation of mesenchymal stem cells augments tendon-like tissue formation and defect repair in vivo. Stem Cells Transl Med 5: 1106-1116, 2016.

8. Hotten GC, Matsumoto T, Kimura M, Bechtold RF, Kron R, Ohara T, Tanaka H, Satoh Y, Okazaki M, Shirai T, et al: Recombinant human growth/differentiation factor 5 stimulates mesenchyme aggregation and chondrogenesis responsible for the skeletal development of limbs. Growth Factors 13: 65-74, 1996.

9. Rickert M, Wang H, Wieloch P, Lorenz H, Steck E, Sabo D and Richter W: Adenovirus-mediated gene transfer of growth and differentiation factor-5 into tenocytes and the healing rat Achilles tendon. Connect Tissue Res 46: 175-183, 2005.

10. Shen H, Gelberman RH, Silva MJ, Sakiyama-Elbert SE and Thomopoulos S: BMP12 induces tenogenic differentiation of adipose-derived stromal cells. PLoS One 8: e77613, 2013.

11. Nochi H, Sung JH, Lou J, Adkisson HD, Maloney WJ and Hruska KA: Adenovirus mediated BMP-13 gene transfer induces chondrogenic differentiation of murine mesenchymal progenitor cells. J Bone Miner Res 19: 111-122, 2004.

12. Rickert M: BMP-14 gene therapy increases tendon tensile strength in a rat model of achilles tendon injury. J Bone Joint Surg Am 90: 445-446, 2008

13. Ozasa Y, Gingery A, Thoreson AR, An KN, Zhao C and Amadio PC: A comparative study of the effects of growth and differentiation factor 5 on muscle-derived stem cells and bone marrow stromal cells in an in vitro tendon healing model. J Hand Surg Am 39: 1706-1713, 2014

14. Hayashi M, Zhao C, An KN and Amadio PC: The effects of growth and differentiation factor 5 on bone marrow stromal cell transplants in an in vitro tendon healing model. J Hand Surg Eur Vol 36: 271-279, 2011.

15. Qu Y, Zhang J, Wu S, Li B, Liu S and Cheng J: SIRT1 promotes proliferation and inhibits apoptosis of human malignant glioma cell lines. Neurosci Lett 525: 168-172, 2012.

16. Takeda-Watanabe A, Kitada M, Kanasaki K and Koya D: SIRT1 inactivation induces inflammation through the dysregulation of autophagy in human THP-1 cells. Biochem Biophys Res Commun 427: 191-196, 2012

17. Park SY, Lee SW, Kim HY, Lee SY, Lee WS, Hong KW and Kim CD: SIRT1 inhibits differentiation of monocytes to macrophages: Amelioration of synovial inflammation in rheumatoid arthritis. J Mol Med (Berl) 94: 921-931, 2016

18. Buhrmann C, Busch F, Shayan P and Shakibaei M: Sirtuin-1 (SIRT1) is required for promoting chondrogenic differentiation of mesenchymal stem cells. J Biol Chem 289: 22048-22062, 2014

19. Joe IS, Jeong SG and Cho GW: Resveratrol-induced SIRT1 activation promotes neuronal differentiation of human bone marrow mesenchymal stem cells. Neurosci Lett 584: 97-102, 2015.

20. Chen Q, Lu H and Yang H: Chitosan prevents adhesion during rabbit flexor tendon repair via the sirtuin 1 signaling pathway. Mol Med Rep 12: 4598-4603, 2015.

21. Cao C, Lu S, Kivlin R, Wallin B, Card E, Bagdasarian A, Tamakloe T, Wang WJ, Song X, Chu WM, et al: SIRT1 confers protection against UVB- and $\mathrm{H} 2 \mathrm{O} 2$-induced cell death via modulation of p53 and JNK in cultured skin keratinocytes. J Cell Mol Med 13: 3632-3643, 2009.

22. Zerr P, Palumbo-Zerr K, Huang J, Tomcik M, Sumova B, Distler O, Schett G and Distler JH: Sirt1 regulates canonical TGF- $\beta$ signalling to control fibroblast activation and tissue fibrosis. Ann Rheum Dis 75: 226-233, 2016.

23. Chen W, Zhang L, Shao SX, Wang HP, Cui SJ, Zhang YN Kong XZ, Yin Q and Zhang JP: Transcription factors GATA4 and TBX5 promote cardiomyogenic differentiation of rat bone marrow mesenchymal stromal cells. Histol Histopathol 30 $1487-1498,2015$
24. Li ZW, Piao CD, Sun HH, Ren XS and Bai YS: Asiatic acid inhibits adipogenic differentiation of bone marrow stromal cells. Cell Biochem Biophys 68: 437-442, 2014

25. Modica $S$ and Moschetta A: Nuclear bile acid receptor FXR as pharmacological target: Are we there yet? FEBS Lett 580 5492-5499, 2006.

26. Id Boufker H, Lagneaux L, Fayyad-Kazan H, Badran B, Najar M, Wiedig M, Ghanem G, Laurent G, Body JJ and Journé F: Role of farnesoid X receptor (FXR) in the process of differentiation of bone marrow stromal cells into osteoblasts. Bone 49: 1219-1231, 2011.

27. Evans RM: The steroid and thyroid hormone receptor superfamily. Science 240: 889-895, 1988.

28. Jia S, Liu X, Li W, Xie J, Yang L and Li L: Peroxisome proliferator-activated receptor gamma negatively regulates the differentiation of bone marrow-derived mesenchymal stem cells toward myofibroblasts in liver fibrogenesis. Cell Physiol Biochem 37: 2085-2100, 2015.

29. Evans JF, Rodriguez S and Ragolia L: ACTH promotes chondrogenic nodule formation and induces transient elevations in intracellular calcium in rat bone marrow cell cultures via MC2-R signaling. Cell Tissue Res 352: 413-425, 2013.

30. Jin M, Chen Y, Zhou Y, Mei Y, Liu W, Pan C and Hua X: Transplantation of bone marrow-derived mesenchymal stem cells expressing elastin alleviates pelvic floor dysfunction. Stem Cell Res Ther 7: 51, 2016.

31. Andersen CL, Jensen JL and Ørntoft TF: Normalization of real-time quantitative reverse transcription-PCR data: A model-based variance estimation approach to identify genes suited for normalization, applied to bladder and colon cancer data sets. Cancer Res 64: 5245-5250, 2004.

32. Méndez-Ferrer S, Michurina TV, Ferraro F, Mazloom AR, Macarthur BD, Lira SA, Scadden DT, Ma'ayan A, Enikolopov GN and Frenette PS: Mesenchymal and haematopoietic stem cells form a unique bone marrow niche. Nature 466: 829-834, 2010

33. Guo KT, SchAfer R, Paul A, Gerber A, Ziemer G and Wendel HP: A new technique for the isolation and surface immobilization of mesenchymal stem cells from whole bone marrow using high-specific DNA aptamers. Stem Cells 24: 2220-2231, 2006.

34. Schweitzer R, Chyung JH, Murtaugh LC, Brent AE, Rosen V, Olson EN, Lassar A and Tabin CJ: Analysis of the tendon cell fate using Scleraxis, a specific marker for tendons and ligaments. Development 128: 3855-3866, 2001.

35. Shukunami C, Takimoto A, Oro M and Hiraki Y: Scleraxis positively regulates the expression of tenomodulin, a differentiation marker of tenocytes. Dev Biol 298: 234-247, 2006.

36. Docheva D, Hunziker EB, Fässler R and Brandau O: Tenomodulin is necessary for tenocyte proliferation and tendon maturation. Mol Cell Biol 25: 699-705, 2005

37. Muraglia A, Cancedda R and Quarto R: Clonal mesenchymal progenitors from human bone marrow differentiate in vitro according to a hierarchical model. J Cell Sci 113: 1161-1166, 2000.

38. Qiang L, Wang L, Kon N, Zhao W, Lee S, Zhang Y, Rosenbaum M, Zhao Y, Gu W, Farmer SR and Accili D: Brown remodeling of white adipose tissue by SirT1-dependent deacetylation of Ppar $\gamma$. Cell 150: 620-632, 2012.

39. Yoshida Y, Tanaka S, Umemori H, Minowa O, Usui M, Ikematsu N, Hosoda E, Imamura T, Kuno J, Yamashita T, et al: Negative regulation of BMP/Smad signaling by Tob in osteoblasts. Cell 103: 1085-1097, 2000.

40. Celil AB and Campbell PG: BMP-2 and insulin-like growth factor-I mediate Osterix (Osx) expression in human mesenchymal stem cells via the MAPK and protein kinase D signaling pathways. J Biol Chem 280: 31353-31359, 2005.

41. Rodeo SA, Sugiguchi F, Fortier LA, Cunningham ME and Maher S: What's new in orthopaedic research. J Bone Joint Surg Am 96: 2015-2019, 2014.

42. Kan A, Ikeda T, Fukai A, Nakagawa T, Nakamura K, Chung UI, Kawaguchi $\mathrm{H}$ and Tabin CJ: SOX11 contributes to the regulation of GDF5 in joint maintenance. BMC Dev Biol 13: 4, 2013.

43. Kadomatsu H, Matsuyama T, Yoshimoto T, Negishi Y, Sekiya H, Yamamoto $\mathbf{M}$ and Izumi Y: Injectable growth/differentiation factor-5-recombinant human collagen composite induces endochondral ossification via Sry-related HMG box 9 (Sox9) expression and angiogenesis in murine calvariae. J Periodontal Res 43: 483-489, 2008.

44. Oshin AO, Caporali E, Byron CR, Stewart AA and Stewart MC: Phenotypic maintenance of articular chondrocytes in vitro requires BMP activity. Vet Comp Orthop Traumatol 20: 185-191, 2007. 
45. Aspenberg P: Stimulation of tendon repair: Mechanical loading, GDFs and platelets. A mini-review. Int Orthop 31: 783-789, 2007.

46. Hinoi E, Nakamura Y, Takada S, Fujita H, Iezaki T, Hashizume S, Takahashi S, Odaka Y, Watanabe T and Yoneda Y: Growth differentiation factor-5 promotes brown adipogenesis in systemic energy expenditure. Diabetes 63: 162-175, 2014.

47. Guzman A, Zelman-Femiak M, Boergermann JH Paschkowsky S, Kreuzaler PA, Fratzl P, Harms GS and Knaus P: SMAD versus non-SMAD signaling is determined by latera mobility of bone morphogenetic protein (BMP) receptors. J Biol Chem 287: 39492-39504, 2012.

48. Sieber C, Kopf J, Hiepen KC and Knaus P: Recent advances in BMP receptor signaling. Cytokine \& Growth Factor Reviews 20 : 343-355, 2009.

49. Becatti M, Fiorillo C, Barygina V, Cecchi C, Lotti T, Prignano F, Silvestro A, Nassi P and Taddei N: SIRT1 regulates MAPK pathways in vitiligo skin: Insight into the molecular pathways of cell survival. J Cell Mol Med 18: 514-529, 2014.

50. Gao P, Xu TT, Lu J, Li L, Xu J, Hao DL, Chen HZ and Liu DP: Overexpression of SIRT1 in vascular smooth muscle cells attenuates angiotensin II-induced vascular remodeling and hypertension in mice. J Mol Med (Berl) 92: 347-357, 2014.

51. Wang H, Kroeber M, Hanke M, Ries R, Schmid C, Poller W and Richter W: Release of active and depot GDF-5 after adenovirus-mediated overexpression stimulates rabbit and human intervertebral disc cells. J Mol Med (Berl) 82: 126-134, 2004.
52. Cucchiarini M and Madry H: Overexpression of human IGF-I via direct $\mathrm{rAAV}$-mediated gene transfer improves the early repair of articular cartilage defects in vivo. Gene Ther 21: 811-819, 2014.

53. Tekari A, Luginbuehl R, Hofstetter W and Egli RJ: Transforming growth factor beta signaling is essential for the autonomous formation of cartilage-like tissue by expanded chondrocytes. PLoS One 10: e0120857, 2015.

54. Murphy MK, Huey DJ, Hu JC and Athanasiou KA: TGF- $\beta 1$, GDF-5, and BMP-2 stimulation induces chondrogenesis in expanded human articular chondrocytes and marrow-derived stromal cells. Stem Cells 33: 762-773, 2015.

55. Solursh M: Cell-cell interactions and chondrogenesis. In: Cartilage: Development, Differentiation, and Growth. Hall BK (ed). Vol 2. Academic Press, New York, NY, pp121-141, 1983.

This work is licensed under a Creative Commons Attribution-NonCommercial-NoDerivatives 4.0 International (CC BY-NC-ND 4.0) License. 